\title{
The Structure Factors of Liquid Metals in Low $Q$ Region
}

\author{
Y. Waseda \\ Research Institute of Mineral Dressing and Metallurgy (SENKEN), Tohoku University, \\ Sendai 980, Japan and \\ Department of Materials Science and Engineering, University of Pennsylvania, Philadelphia, \\ Pennsylvania 19104, USA
}

\section{Z. Naturforsch. 38 a, 509-515 (1983); received November 26, 1982}

The low $Q$ structure factors have been determined in the region down to $Q=0.08 \mathrm{~A}^{-1}$ for liquid $\mathrm{Na}, \mathrm{K}, \mathrm{Rb}, \mathrm{Cs}, \mathrm{Mg}, \mathrm{Ca}, \mathrm{Sr}, \mathrm{Ba}, \mathrm{Al}$ and $\mathrm{In}$ at several temperatures by $\mathrm{x}$-ray diffraction with the transmission mode. The low $Q$ structure factor is a slowly increasing function of $Q$, and its profile is quite similar among the liquid metals presently investigated. The long wavelength limit value of $S(Q)$ derived from the present experimental structural data agrees well with that calculated from the isothermal compressibility for seven metals near the melting point. A difference larger than $10 \%$ was found in the cases of liquid $\mathrm{Cs}, \mathrm{Ca}$ and $\mathrm{Al}$.

\section{Introduction}

$\mathrm{X}$-ray and neutron diffraction studies provide a large amount of valuable information about the atomic scale structure of liquid metals [1, 2]. In these previous studies, the determination of the structure factor, $S(Q)$, in high $Q$ region was of primary importance in order to obtain a radial distribution function. On the other hand, the need for structural data concerning the low $Q$ region has been frequently emphasized as a rigorous test of the electron transport theory and the approximate statistical theory for liquid metals (for example, Greenfield [3], North et al. [4], Ballentine and Jones [15]). However, such low $Q$ structure factors are available for only a few metallic elements [6-9]. The low $Q$ behaviour of the structure factor, $S(Q)$, for liquid metals is again recognized to be of considerable interest, in parallel with recent theoretical progress in liquid metals (Evans and Sluckin [10], Ohkoshi et al. [11], McLaughlin and Young [12], Matthai and March [13]). With this fact in mind, the main purpose of this work is to provide information on the low $Q$ structure factors of liquid metals at various temperatures carefully and systematically measured by $\mathrm{x}$-ray diffraction.

\section{Experimental Procedure}

Following the detailed discussions and results given in previous works $[7-9,14-15]$ a transmis-

Reprint requests to Professor Y. Waseda, Department of Materials Science and Engineering K1, University of Pennsylvania, Philadelphia, PA 19104, USA. sion mode with $\mathrm{MoK}_{\alpha}$ and $\mathrm{Rh}-\mathrm{K}_{\alpha}$ radiation was used in this work. The basic experimental arrangements and operating procedures were almost identical to those employed in the previous works [7,9] and are therefore not duplicated here. Only some relevant details are given below.

The samples were prepared from the following materials with nominal purity indicated in \%; $\mathrm{Na}$ (99.93), K (99.99), Rb (99.95), Cs (99.98), Mg (99.9), $\mathrm{Ca}$ (99.0), Sr (99.0), Ba (99.0), Al (99.99) and In (99.999). A line $\mathrm{x}$-ray source is monochromatized with a bent and ground LiF crystal in a primary beam whose focal spot lies beyond the sample and just before the detector. The slit system is as follows; divergent slit: $1^{\circ}$, receiving slit $0.1 \mathrm{~mm}$ and scattered slit: $1 \mathrm{~mm}$. The sample table of the horizontal diffractometer rotates at half the angle of the detector arm to maintain the focusing condition. Most of the data were obtained by Mo- $\mathrm{K}_{\alpha}$ radiation, but the measurement with $\mathrm{Rh}-\mathrm{K}_{\alpha}$ radiation was also used so as to avoid the well-known disadvantages such as the fact that the energies of $\mathrm{Rb}-\mathrm{K}_{\beta}$ and $\mathrm{Sr}$ $\mathrm{K}_{\beta}$ are close to that of $\mathrm{Mo}-\mathrm{K}_{\alpha}$. The sample was placed in the high temperature sample holder made of $18 / 8$ stainless steel, the basic design of which is very similar to that employed by Huijben and van der Lugt [9], and heated with a spiral SYTT(Fe$25 \mathrm{wt} \% \mathrm{Cr}-4 \mathrm{wt} \% \mathrm{Al}-1 \mathrm{wt} \% \mathrm{Y}$ ) heating element to the desired temperature within $\pm 1 \mathrm{deg}$. Depending on temperature and element, a beryllium sheet, $0.12 \mathrm{~mm}$ thick, or a pyrolitic graphite sheet, $0.15 \mathrm{~mm}$ thick, was used as a window material with boron nitride supports. A very thin $(10 \mu \mathrm{m})$ nickel or tantalum foil 
was also used to protect the beryllium window from attack by the chemically reactive elements such as heavy alkaline earth metals. The sample thickness was continuously varied by mechanically moving the windows. The spacing of two window materials is adjustable between $0.03 \mathrm{~mm}$ and $3 \mathrm{~mm}$. This high temperature sample holder was enclosed under $\mathrm{He}$ atmosphere in a container with thin Mylar windows.

Two $x$-ray scattering measurements were carried out for each sample; one is to obtain the intensity from the empty cell and the other is from the cell containing the sample. A brief description for the correction of the observed intensity data is also given below, although these procedures are now very common. The polarization correction for a mosaic crystal has been applied in this analysis. The product of absorption coefficient and sample thickness was determined experimentally as a function of angles at every temperature presently investigated. The correction for the Compton scattering was based on a calculation with the values reported by Cromer and Mann [16] and the so-called Breit-Dirac recoil factor (see, e.g. Wagner [17]). The small wavelength dependence of the Compton scattering intensity, which is mainly the results of the slightly different linear absorption coefficient after scattering, was also considered using Victoreen's formula [18] in this work. The evaluation of the Compton scattering component in the measured intensity data was independently checked by measurements using a Ge-SSD with a very narrow window, or by using a diffracted monochromator mode coupled with the socalled Ruland method [17]. The multiple scattering correction was considered only for the doubly scattered radiations along the lines proposed by Malet et al. [19]. These correcting procedures are almost identical to those employed in the previous works on liquid alkali metals and tin [7,9] and have already been described in detail. It may be worth mentioning that these corrections appear not to be critically important for the metallic elements presently investigated. For normalization, the generaliized Krogh-Moe-Norman's method was used in conjunction with the atomic scattering factor calculated by Cromer and Waber [20] together with the anomalous dispersion correction factor [21]. The method proposed by Rahman [22] was also employed for checking the normalization with the measured density values, which are known with an uncertainty of $0.4-0.7 \%$.

\section{Results and Discussion}

The low $Q$ structure factors were evaluated in the following way. In the first step, the conventional experiment of transmission mode (hereafter to be referred to as large angle measurements) was done to obtain the structure factor $S(Q)$ as a function of $Q$ with emphasis on the region larger than $0.5 \mathrm{~A}^{-1}$. The results are given in Fig. 1 for liquid $\mathrm{Na}$ at $105^{\circ} \mathrm{C}$ as an example. The increase in the value of $S(Q)$ below $Q=0.5 \mathrm{~A}^{-1}$ is a well-known spurious effect related to the sensing of the primary beam by the detector system. This effect has partially been overcome by placing an additional slit system [23] which more narrowly defines the incident and diffracted beams. In the second step, a more accurate low angle measurement was carried out using a very narrow beam. The readjustment of the slit system was also employed in this measurement to penetrate sufficiently deep into the liquid sample in the low angle region. Such readjustment has been applied by Huijben and van der Lugt [9]. The low angle results are superimposed on the large angle results obtained in the first step as shown in Fig. 1 using the results of liquid $\mathrm{Na}$ at $105^{\circ} \mathrm{C}$ as an example. The uncertainty in the normalization of the structure factor calculated from the results in the low angle measurements with the narrow beam is reduced when overlapping results are obtained in the region of the first peak using two measurements (low angle and high angle measurements). In the previous works of North and Wagner [7] and Green-

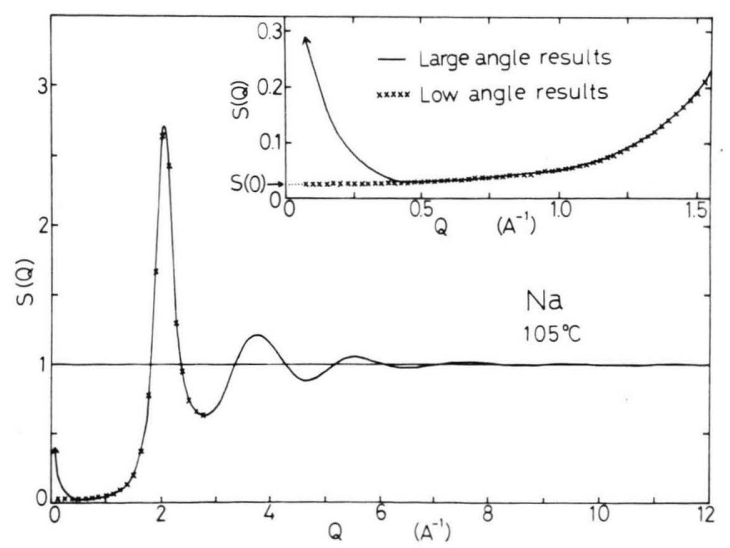

Fig. 1. Structure factor $S(Q)$ of liquid $\mathrm{Na}$ at $105^{\circ} \mathrm{C}$. The solid line denotes the results of large angle scattering measurements and the crosses are the results of low angle scattering measurements. The insert shows the values in the low $Q$ region. 
field et al. [8], only a single measurement was used to determine the low $Q$ structure factor, whereas the superimposition of two data was made in the limited $Q$ region between 0.2 and $0.5 \mathrm{~A}^{-1}$ in the work of Huijben and van der Lugt [9]. By the present technique of two different measurements, it was possible to obtain more accurate information down to a value of $Q=0.08 \mathrm{~A}^{-1}$.

The experimental uncertainty in the low angle results themselves is difficult to estimate. However, the following points are suggested. The accumulated counts varying from $6 \times 10^{4}\left(Q \leqq 5.0 \mathrm{~A}^{-1}\right)$ in the low angle measurements to $2 \times 10^{5}$ $\left(Q>5.0 \mathrm{~A}^{-1}\right)$ in the high angle measurements were chosen so that the counting statistics were approximately uniform. On the basis of the check by Rahman's method, the normalization for the present large angle measurements is in error by less than $1.8 \%$. A source of systematic errors for the liquid structure factors by $\mathrm{x}$-ray diffraction arises from the uncertainties of the atomic scattering factor, of the Compton scattering and of the multiple scattering. According to the detailed discussion given by Greenfield et al. [8] and Marlet et al. [9], the maximum error in these quantities seems not to exceed $1 \%$ for the metals presently investigated. Therefore, the total uncertainty of the structure factors obtained from the large angle measurements in this work is estimated to be $2.8 \%$. The mismatch in the superimposition of two x-ray scattering intensities (low angle and high angle measurements) was of the order of $0.6 \%$ over the range of $Q$ presently investigated. Thus, an uncertainty of $3.4 \%$ in the low $Q$ structure factors in this work may be suggested from these uncertainties including the counting statistics in the low angle measurements. We could also suggest that the relative changes $\delta S(Q) / \delta T$ arising from the temperature variation are in error by less than $1 \%$, i.e. the accuracy of the temperature dependence of the low $Q$ structure factors obtained in this work is higher than that of the structure factor itself.

The experimental results indicate a smooth variation of the low $Q$ structure factor $S(Q)$ as a function of $Q$ for ten liquid metals at several temperatures presently investigated. Such structural feature is exemplified by Fig. 2 using the results of liquid $\mathrm{Na}$ as an example. The low $Q$ structure factor gives a slowly increasing function of $Q$, particularly in the range of $Q \leqq Q_{1} / 2$ where $Q_{1}$ is the first peak posi-

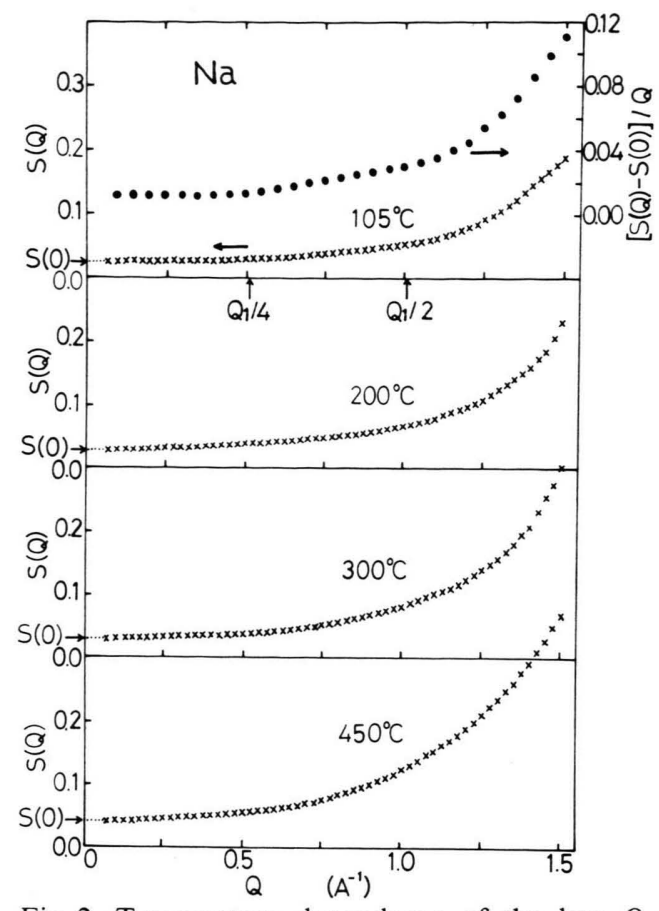

Fig. 2. Temperature dependence of the low $Q$ structure factor $S(Q)$ of liquid Na. The crosses denote the plot of $[S(Q)-S(0)] / Q$ as a function of $Q$.

tion in the structure factor, while it rises rapidly to its peak value for $Q>Q_{1} / 2$. We also note the following two points: (1) The absolute values of low $Q$ structure factors increase when the temperature is raised. (2) The low $Q$ structure factor at temperatures close to the melting point gives a nearly constant value in the $Q$ range less than $Q_{1} / 4$.

The present experimental results suggest that the low $Q$ structure factor $S(Q)$ of liquid metals for $Q \leqq Q_{1} / 2$ can be expressed in the following polynomial form:

$$
S(Q)=a_{0}+a_{1} Q+a_{2} Q^{2},
$$

where $a_{0}, a_{1}$ and $a_{2}$ are the coefficients. The importance of the linear term of $a_{1} Q$ in (1) has recently been stressed for liquid metals by Matthai and March [13]. In addition, the higher terms such as $a_{3} Q^{3}$ and $a_{4} Q^{4}$ are, of course, required for fitting the measured structure factor in a wider $Q$ region. As easily seen from the results of Fig. 2, the present experimental data can be extrapolated smoothly to the long wavelength value of $S(0)$ calculated from the well-known relation;

$$
S(0)=\varrho_{0} k_{\mathrm{B}} T \chi_{\mathrm{T}},
$$


where $\varrho_{0}$ is the number density of atoms, $k_{\mathrm{B}}$ is the Boltzmann constant, $T$ is the absolute temperature and $\chi_{T}$ is the isothermal compressibility [24]. Thus the coefficient $a_{0}$ in (1) is often replaced by the $S(0)$ value. However, it should be kept in mind that the value of $S(0)$ obtained from Eq. (2) is subject to some ambiguity (about $10 \%$ ), part arising from the uncertainty in the isothermal compressibility value as was already mentioned by Greenfield et al. [8]. For this reason, the value of $a_{0}=S(0)$ was evaluated in this work directly from the measured low $Q$ structure factor by the following manner.

In the $Q$ range less than $Q_{1} / 4$, the following simplification can be made on the basis of experimental data;

$$
S(Q)=S(0)+a_{1} Q\left(Q \leqq Q_{1} / 4\right) .
$$

This is confirmed by the plot of $[S(Q)-S(0)] / Q$ as a function of $Q$ as shown in the case of liquid $\mathrm{Na}$ at $105^{\circ} \mathrm{C}$ in Fig. 2, where $S(0)$ value of 0.0234 calculated from the isothermal compressibility data of Webber and Stephens [25] was used. With this experimental fact in mind, (3) can be rewritten in the following form;

$$
\frac{S(Q)}{Q}=\frac{S(0)}{Q}+a_{1} \quad\left(Q \leqq Q_{1} / 4\right) .
$$

Then, one obtain, with high accuracy, the values of $S(0)$ and possibly $a_{1}$ from the plot of $S(Q) / Q$ as a function of $1 / Q$ in the low $Q$ region such as $Q \leqq Q_{1} / 4$. The coefficient $a_{1}$ should be determined by using the structure factor in a wider $Q$ region, so that our attention in this process focuses only to the determination of the $S(0)$ value directly from the measured low $Q$ structure factor.

Figure 3 gives the plot of $S(Q) / Q$ against $1 / Q$ for liquid $\mathrm{Na}$ at several temperatures. The plotted points are the experimental data and the straight lines are the fit to the form of (4). The results of Fig. 3 clearly show that it is possible to determine the $S(0)$ value from the measured structural data alone using the formula (4). A similar feature was also obtained in other liquid metals as shown in Fig. 4. The $S(0)$ values obtained in this procedure for ten liquid metals at several temperatures are summarized in Table 1 together with the corresponding values derived from the isothermal compressibility data using (2). The overall agreement between $S(0)$ and $\varrho_{0} k_{\mathrm{B}} T \chi_{T}$ is rather surprisingly good, although the difference exceeds $10 \%$ in the

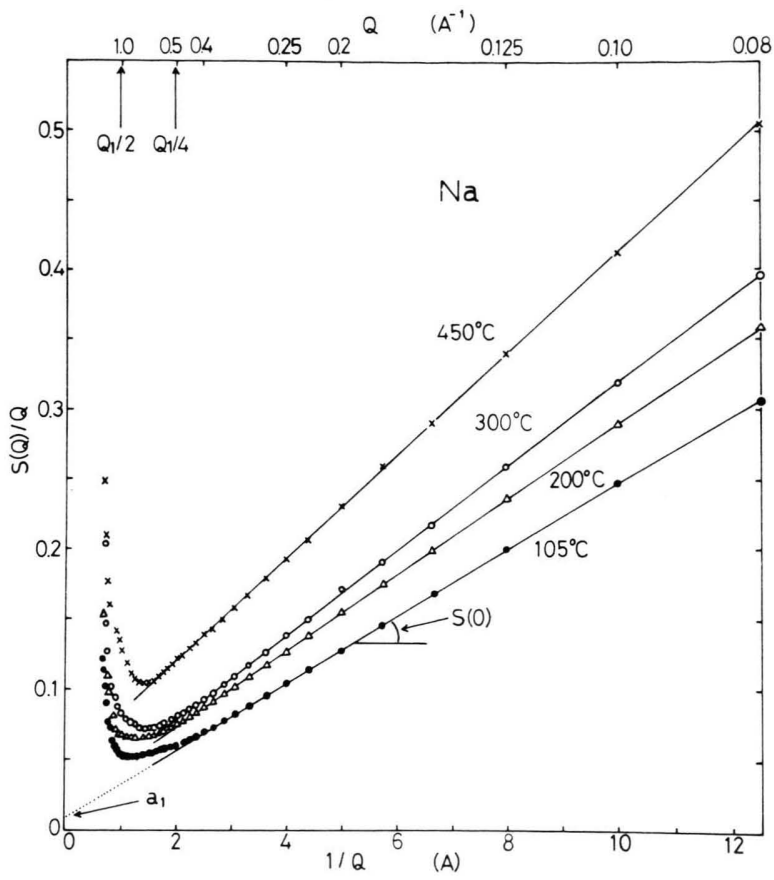

Fig. 3. Relationship between $S(Q) / Q$ and $1 / Q$ for liquid $\mathrm{Na}$ at several temperatures. The plotted points are the experimental data and the straight lines are the fit to the form of (4).

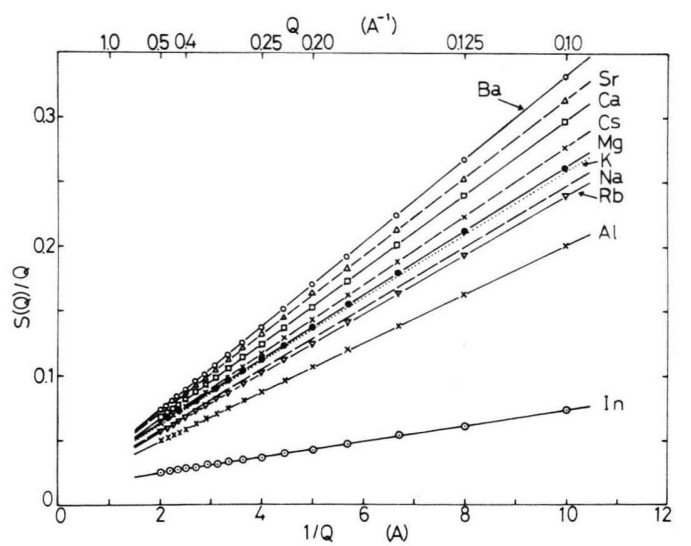

Fig. 4. Relationships between $S(Q) / Q$ and $1 / Q$ for various liquid metals near the melting point in the $Q$ region less than $0.5 \mathrm{~A}^{-1}$. The plotted points are the experimental data and the straight lines are the fit to the form of (4).

cases of liquid $\mathrm{Cs}, \mathrm{Ca}$ and $\mathrm{Al}$. On the basis of the atomic level stresses in disordered system, Egami and Srolovitz [26] recently suggest that the microscopic isothermal compressibility related to the local fluctuation in liquids is not necessarily equal to the macroscopic isothermal compressibility. Such 
Table 1. Comparison of the long wavelength value of $S(0)$ obtained from diffraction data with those calculated from the isothermal compressibility.

\begin{tabular}{|c|c|c|c|c|c|c|}
\hline \multicolumn{2}{|c|}{$\begin{array}{l}\text { Temp. } \\
\left({ }^{\circ} \mathrm{C}\right) \cdot\end{array}$} & $\begin{array}{l}\text { Density } \\
\left(\mathrm{g} / \mathrm{cm}^{3}\right)\end{array}$ & $\begin{array}{l}\chi_{T} \\
\times 10^{-12}\left(\mathrm{~cm}^{2} / \mathrm{dyn}\right)\end{array}$ & $\varrho_{0} k_{\mathrm{B}} T \chi_{T}$ & $\begin{array}{l}S(0) \\
\text { (Present } \\
\text { work) }\end{array}$ & $\frac{\varrho_{0} k_{\mathrm{B}} T \chi_{T}-S(0)}{\varrho_{0} k_{\mathrm{B}} T \chi_{T}}$ \\
\hline & $\begin{array}{l}105 \\
200 \\
300 \\
450\end{array}$ & $\begin{array}{l}0.928 \\
0.903 \\
0.881 \\
0.847\end{array}$ & $18.6[25]$ & 0.0234 & $\begin{array}{l}0.023 \\
0.028 \\
0.032 \\
0.037\end{array}$ & +1.7 \\
\hline $\mathrm{K}$ & $\begin{array}{r}70 \\
105 \\
200 \\
350\end{array}$ & $\begin{array}{l}0.826 \\
0.815 \\
0.797 \\
0.763\end{array}$ & $38.2[25]$ & 0.0231 & $\begin{array}{l}0.024 \\
0.025 \\
0.032 \\
0.035\end{array}$ & -3.9 \\
\hline $\mathrm{Rb}$ & $\begin{array}{r}40 \\
100 \\
200\end{array}$ & $\begin{array}{l}1.416 \\
1.448 \\
1.397\end{array}$ & $49.3[25]$ & 0.0221 & $\begin{array}{l}0.022 \\
0.025 \\
0.033\end{array}$ & +0.5 \\
\hline Cs & $\begin{array}{r}30 \\
100 \\
200\end{array}$ & $\begin{array}{l}1.838 \\
1.796 \\
1.739\end{array}$ & $68.8[25]$ & 0.0239 & $\begin{array}{l}0.027 \\
0.032 \\
0.037\end{array}$ & -13.0 \\
\hline $\mathrm{Mg}$ & $\begin{array}{l}680 \\
790 \\
880\end{array}$ & $\begin{array}{l}1.545 \\
1.433 \\
1.339\end{array}$ & $5.06[27]$ & 0.0255 & $\begin{array}{l}0.025 \\
0.028 \\
0.031\end{array}$ & +2.0 \\
\hline $\begin{array}{l}\mathrm{Ca} \\
\mathrm{Sr} \\
\mathrm{Ba}\end{array}$ & $\begin{array}{l}850 \\
780 \\
730\end{array}$ & $\begin{array}{l}1.37 \\
2.38 \\
3.32\end{array}$ & $\begin{array}{l}11.0[27] \\
13.1[27] \\
17.9[27]\end{array}$ & $\begin{array}{l}0.0349 \\
0.0310 \\
0.0362\end{array}$ & $\begin{array}{l}0.031 \\
0.032 \\
0.035\end{array}$ & $\begin{array}{l}+11.2 \\
-\quad 3.2 \\
+3.3\end{array}$ \\
\hline $\mathrm{Al}$ & $\begin{array}{r}670 \\
750 \\
1050\end{array}$ & $\begin{array}{l}2.37 \\
2.35 \\
2.27\end{array}$ & $2.42[28]$ & 0.0166 & $\begin{array}{l}0.019 \\
0.021 \\
0.024\end{array}$ & -14.5 \\
\hline In & $\begin{array}{l}160 \\
300 \\
500 \\
700\end{array}$ & $\begin{array}{l}7.03 \\
6.92 \\
6.77 \\
6.61\end{array}$ & 2.96 [29] & 0.0065 & $\begin{array}{l}0.0068 \\
0.0098 \\
0.014 \\
0.020\end{array}$ & -4.6 \\
\hline
\end{tabular}

situation may account for part of the difference, as well as the experimental uncertainties in both $S(0)$ and $\chi_{T}$. However, the origin of the difference between $S(0)$ and $\varrho_{0} k_{\mathrm{B}} T \chi_{T}$ of liquid $\mathrm{Cs}, \mathrm{Ca}$ and $\mathrm{Al}$ cannot be clearly identified at the present time.

The structural features emerging from the present low $Q$ structure factors are consistent with the previous conclusion which has been obtained for some liquid metals [6-13] from the standpoints of both theory and experiments. A shallow minimum in the low $Q$ structure factor of liquid alkali metals predicted by the second order pseudopotential theory of metals (Evans and Sluckin [10]) was not observed by us. However, we may add that a very weak but slowly decreasing tendency of the intensity pattern as a function of $Q$ was detected in the case of liquid $\mathrm{Na}$ at $610^{\circ} \mathrm{C}$ as shown in Figure 5 . Although the profile of the difference in the $\mathrm{x}$-ray scattering intensity data between $550^{\circ} \mathrm{C}$ and $610^{\circ} \mathrm{C}$,

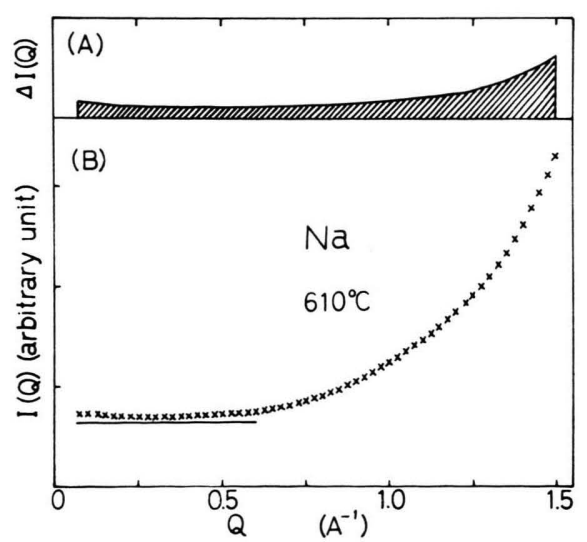

Fig. 5. The low $Q$ x-ray scattering intensity $I(Q)$ of liquid $\mathrm{Na}$ at $610^{\circ} \mathrm{C} . \Delta I(Q)$ corresponds to the temperature difference of intensity data between $550^{\circ} \mathrm{C}$ and $610^{\circ} \mathrm{C}$ normalized to the first peak height. The quantitative accuracy is not the same as those of other measurements given in Figs. $1-4$ due to the experimental difficulties. 

Table 2. Parameters of polynomial function for the low $Q$ structure factors determined in this work. $S(Q)=\sum_{i=0} \alpha_{i} Q^{i},\left(0.08 \leqq Q \leqq 1.0 \mathrm{~A}^{-1}\right)$ and the fitting
uncertainty was less than $0.5 \%$ in most cases.

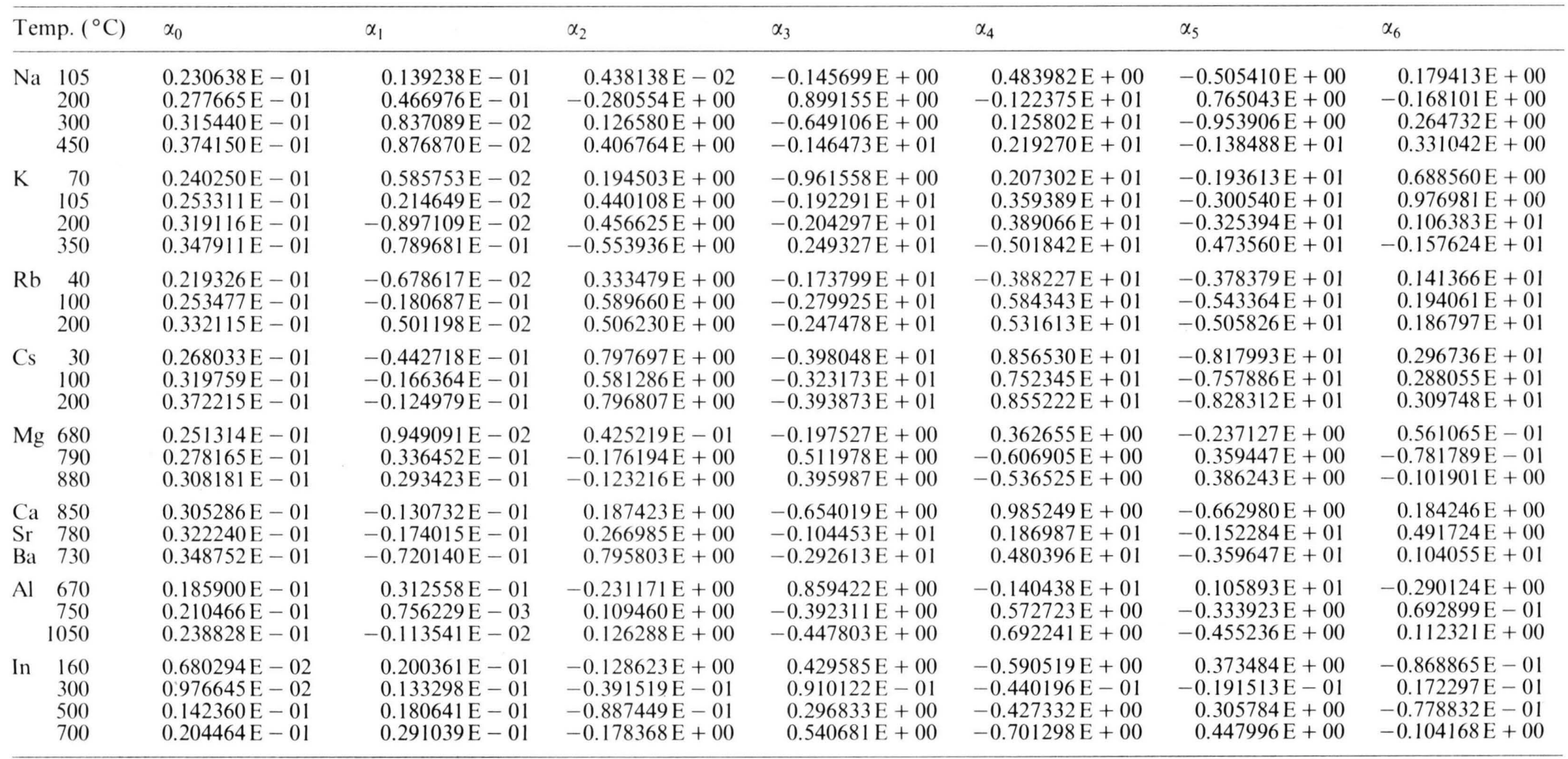


which are normalized to the first peak height (Fig. 5A), indicates a slowly decreasing tendency in the $Q$ region below $0.3 \mathrm{~A}^{-1}$, it should be noted that these relatively high temperature measurements were seriously affected by the experimental difficulties: Mainly due to the active chemical reactivity of liquid sodium metal, the structure factor of liquid $\mathrm{Na}$ at $610^{\circ} \mathrm{C}$ could not have been determined with sufficient accuracy. Therefore, further experimentation at higher temperatures may be required before the full implication of the observed shallow minimum in the low $Q$ structure factor of liquid alkali metals can be assessed.

\section{Acknowledgements}

The author is indebted to Professor T. Egami for reading and commenting on the manuscript. This work was completed during a scholastic leave of the author spent in the Department of Materials Science and Engineering, University of Pennsylvania. During this period, he wishes to acknowledge partial support by the National Science Foundation through DMR81-00405.

[1] J. E. Enderby, Physics of Simple Liquids, edited by N. H. Temperly, J. S. Rowlinson, and G. S. Rushbrooke, North-Holland, Amsterdam 1968, p. 612.

[2] Y. Waseda, The Structure of Non-Crystalline Materials, McGraw-Hill, New York 1980.

[3] A. J. Greenfield, Phys. Lett. 16,6 (1966).

[4] D. M. North, J. E. Enderby, and P. A. Egelstaff, J. Phys. C. 1, 784 (1968).

[5] L. E. Ballentine and J. C. Jones, Can. J. Phys. 5, 1831 (1973).

[6] P. A. Egelstaff, C. Duffill, V. Rainey, J. E. Enderby, and D. M. North, Phys. Lett. 21, 286 (1966).

[7] D. M. North and C. N. J. Wagner, J. Appl. Cryst. 2, 149 (1969).

[8] A. J. Greenfield, J. Wellendorf, and N. Wiser, Phys. Rev. A4, 1607 (1971).

[9] M. J. Huijben and W. van der Lugt, Acta Cryst. A35, 431 (1979).

[10] R. Evans and T. J. Sluckin, J. Phys. C. 14, 3137 (1981).

[11] I. Ohkoshi, I. Yokoyama, Y. Waseda, and W. H. Young, J. Phys. F. 11, 531 (1981).

[12] I. L. McLaughlin and W. H. Young, J. Phys. F. 12, 245 (1982).

[13] C. C. Matthai and N. H. March, Phys. Chem. Liquids 11, 207 (1982).

[14] S. Steeb, H. Dilger, and H. Hoehler, Phys. Chem. Liquids 1, 235 (1969).
Appendix

The large angle results obtained by the transmission mode in this work agree fairly well with those previously reported by the reflection mode [2]. The numerical values of the structure factor in the $Q$ region larger than $0.5 \mathrm{~A}^{-1}$ have already been listed in the previous report [2], although less accuracy of these data may be suggested in the $Q$ region smaller than $Q_{1} / 2\left(\mathrm{~A}^{-1}\right)$, where $Q_{1}$ is the first peak position in the structure factor. The low $Q$ structure factors obtained with sufficient accuracy in this work were expressed by polynomials and the parameters determined by the least-squares fitting procedure are given in Table 2 for the convenience of further quantitative discussion of various properties of liquid metals. However, it should be kept in mind that the physically meaningful origin of the parameters of higher terms cannot be specified and these higher terms were required only for reproducing the measured structure factors in the $Q$ region between 0.08 and $1.0 \mathrm{~A}^{-1}$ with the fitting uncertainty less than $0.5 \%$.

[15] A. Boos, S. Steeb, and D. Godel, Z. Naturforsch. 27 a, 271 (1972).

[16] D. T. Cromer and J. B. Mann, J. Chem. Phys. 47, 1892 (1967).

[17] C. N. J. Wagner, J. Non-Cryst. Solids 31, 1 (1978).

[18] International Tables of Crystallography, Vol. III, Kynoch Press, Birmingham 1962.

[19] G. Malet, C. Cabos, A. Escande, and P. Delord, J. Appl. Cryst. 6, 139 (1973).

[20] D. T. Cromer and J. T. Weber, Acta Cryst. 18, 104 (1965).

[21] D. T. Cromer and D. Libermann, J. Chem. Phys. 53, 1891 (1970).

[22] A. Rahman, J. Chem. Phys. 42, 3540 (1965).

[23] Y. Waseda and S. Tamaki, J. Phys. Soc. Japan 43, 1259 (1977).

[24] L. D. Landau and E. M. Lifschitz, Statistical Physics, Pergamon Press, Oxford 1958.

[25] G. M. B. Webber and R. W. B. Stephens, Physical Acoustics, (Vol. IVB) edited by W. P. Mason, Academic Press, New York 1968, p. 53.

[26] T. Egami and D. Srolovitz, J. Phys. F. 12, 2141 (1982).

[27] S. P. McAlister, E. D. Crozier, and J. F. Cochran, Can. J. Phys. 52, 1847 (1974).

[28] U. Maier and S. Steeb, Phys. and Matter 17, 1 (1973).

[29] J. Hoechler and S. Steeb, Z. Naturforsch. 30a, 784 (1975). 